\title{
Endovascular Reperfusion for Acute Isolated Cervical Carotid Occlusions: The Concept of "Hemodynamic Thrombectomy"
}

\author{
Luís Henrique de Castro-Afonso $^{a} \quad$ Guilherme Seizem Nakiri ${ }^{a}$ \\ Lucas Moretti Monsignore $^{a}$ Francisco Antunes Dias $^{b}$ \\ Frederico Fernandes Aléssio-Alves ${ }^{b}$ Marco Túlio Rezende ${ }^{a}$ \\ Felipe Padovani Trivelato $^{a}$ Octávio Marques Pontes-Neto $^{b}$ \\ Daniel Giansante Abud ${ }^{a}$ \\ aDivision of Interventional Neuroradiology, Ribeirão Preto Medical School, University of \\ São Paulo, Ribeirão Preto, Brazil; ' Division of Neurology, Ribeirão Preto Medical School, \\ University of São Paulo, Ribeirão Preto, Brazil
}

\section{Keywords}

Acute ischemic stroke $\cdot$ Carotid artery occlusion - Carotid stenting · Mechanical thrombectomy

\begin{abstract}
Background/Aims: Endovascular treatment improves the outcomes of patients presenting with acute large vessel occlusions. Isolated proximal carotid occlusions presenting with hemodynamic ischemic stroke may probably also benefit from endovascular treatment. We aimed to assess the clinical and radiological data findings on patients who underwent endovascular treatment for acute ischemic stroke related to an isolated cervical carotid artery occlusion. Methods: Of a consecutive series of 223 patients who were admitted with acute ischemic stroke and were treated by thrombectomy, we included 9 patients with isolated cervical internal carotid occlusions. Results: The mean baseline National Institutes of Health Stroke Scale (NIHSS) score was 11.8. Complete carotid recanalization was achieved in 5 of the 9 patients (55.5\%). In 2 patients, vertebral angioplasty was performed to improve the collateral flow. All patients had a modified Thrombolysis in Cerebral Infarction ( $\mathrm{mTICl}$ ) score of 3 at the end of the procedures. A good neurological outcome, defined as a modified Rankin Scale score $\leq 2$ at the 3 -month follow-up, was observed in 6 patients (66.7\%). No symptomatic intracranial hemorrhages or deaths occurred during the 3 months of follow-up. Conclusions: The endovascular recanalization of isolated cervical carotid occlusions presenting with acute ischemic stroke symptoms is feasible. Because isolated cervical carotid occlusions are associated with hemodynamic ischemic symptoms, if carotid recanalization cannot be achieved,
\end{abstract}


stenting other cervical arteries' stenoses, with a focus on intracranial flow improvement, appears to be a reasonable strategy. Large controlled studies are necessary to assess the safety and efficacy of recanalization of acute isolated cervical carotid occlusions.

(c) 2018 S. Karger AG, Basel

\section{Introduction}

Large vessel occlusions have been reported in approximately $41 \%$ of acute ischemic strokes [1]. Thrombectomy is the standard treatment for acute ischemic stroke due to distal carotid artery or proximal middle cerebral artery occlusions [2-6]. Approximately $15 \%$ of all large vessel occlusion strokes are due to carotid tandem occlusion, which is defined as both an extracranial occlusion, or a stenosis, of the internal carotid artery and a downstream intracranial vessel occlusion. Although recent trials were not designed to evaluate patients presenting with carotid tandem occlusions, a subgroup analysis of the individual data on patients presenting with carotid tandem occlusions suggested that endovascular recanalization results in better clinical outcomes compared to intravenous thrombolysis $[7,8]$. In contrast to carotid tandem occlusions, some patients may present with an acute hemodynamic ischemic stroke due to an isolated cervical carotid occlusion with no associated intracranial occlusion. Acute ischemic stroke due to isolated carotid occlusions is uncommon and seems to also benefit from endovascular treatment $[9,10]$. The role of endovascular treatment in this setting is not completely known and has been poorly reported.

We aimed to assess the clinical and radiological data on patients who underwent endovascular treatment for acute ischemic stroke related to an isolated cervical carotid artery occlusion.

\section{Subjects and Methods}

We evaluated the clinical and radiological data on a consecutive series of patients admitted to our stroke unit with acute ischemic stroke due to isolated cervical internal carotid occlusions who underwent endovascular treatment from June 2011 to June 2017. The patients were selected for endovascular treatment according to our institution's acute stroke protocol for recanalization strategies [11]. Of 223 patients who underwent endovascular treatment for acute stroke due to large vessel occlusions, we excluded 44 patients $(20 \%)$ with vertebrobasilar occlusions, 132 patients (59\%) with distal carotid or M1 occlusions, and 38 patients $(17 \%)$ with carotid tandem occlusions. We included only 9 patients $(4 \%)$ presenting with a cervical carotid occlusion without a downstream intracranial occlusion.

We assessed baseline clinical and radiologic data, defined as age, gender, National Institutes of Health Stroke Scale (NIHSS) scores at admission, baseline modified Rankin Scale (mRS) scores, ASPECTS (Alberta Stroke Program Early CT Score) at baseline and 24 h, etiology of the carotid occlusion, side and extension of the carotid occlusion, collateral contribution to the ischemic territory, recanalization, and procedure times. The primary endpoint was the recanalization rate and the secondary endpoints were symptomatic intracranial hemorrhage (sICH) in hospital and mortality at the 3-month follow-up.

All patients were examined by independent, certified vascular neurologists at hospital admission, who measured their neurological deficits using the NIHSS and mRS. A CT and a CT angiography were done at admission, and a second brain CT scan was obtained after $24 \mathrm{~h}$ of treatment. An isolated cervical internal carotid occlusion was defined as a complete occlusion of the cervical internal carotid and absence of any ipsilateral intracranial occlusion as observed on CT angiography and confirmed by digital subtraction angiography during the procedure. If any ipsilateral intracranial occlusion was observed in a patient, this case was considered a tandem occlusion.

The sICH was classified as primary intracranial hemorrhage type 2 according to the criteria defined by the SITS-MOST study [12]. The procedure time began with groin puncture and ended at the instant of maximal 
recanalization. Recanalization was assessed using the modified Thrombolysis in Cerebral Infarction (mTICI) score. Successful recanalization was defined as a TICI score of 3 or $2 b$ in the carotid territory.

All patients were positioned with the head flat at $0^{\circ}$ and $500-1,000 \mathrm{~mL}$ of saline was intravenously infused. Blood pressure was maintained up to $220 / 120 \mathrm{~mm} \mathrm{Hg}$ if no recombinant tissue plasminogen activator (r-tPA) was infused, and up to 180/105 $\mathrm{mm} \mathrm{Hg}$ if r-tPA was infused.

For the endovascular treatment, intubation of the patients was performed whenever necessary. All of the procedures were performed using a femoral artery access. An intravenous bolus of 5,000 IU of standard heparin was administered after the puncture if intravenous $r$-tPA had not been previously infused. If r-tPA had been infused prior to the endovascular procedure, no heparin was administered. The general antiplatelet regimen that was recommended was aspirin (300 $\mathrm{mg}$ during the procedure) and clopidogrel (75 $\mathrm{mg}$ daily), which was started $24 \mathrm{~h}$ after treatment.

A 7-Fr guiding catheter (Guider Softip; Boston Scientific, Natick, MA, USA) or a 6-Fr guiding sheath (Destination; Terumo, The Netherlands) was introduced through a femoral sheath into the common carotid artery. If a proximal carotid or vertebral occlusion was identified, an angioplasty stenting using a Wallstent or Express stent (both by Boston Scientific Target, Fremont, CA, USA), respectively, was performed. The angioplasty balloons used for predilatation and postdilatation were 2.0, 3.0, 4.0, 5.0, 6.0, or $7.0 \times 20 \mathrm{~mm}$, 153-cm, Monorail angioplasty balloons (Boston Scientific, Maple Grove, MN, USA). In cases in which carotid recanalization was not achieved and patients presented with associated vertebral artery stenosis, defined as a stenosis $\geq 50 \%$, an angioplasty stenting of vertebral arteries was performed, aiming to improve intracranial collateral circulation to the ischemic carotid territory.

Categorical variables are presented as numbers and percentages. Continuous variables are presented as mean \pm SD (range) or median (range). The Statistical Package for the Social Sciences software version 20.0 (SPSS, Chicago, IL, USA) was used for statistical analysis.

\section{Results}

Among the 9 patients included in the study, 5 were men (55.5\%), 8 (88.8\%) had a history of high blood pressure, 2 had diabetes mellitus (22\%), and $6(66.6 \%)$ were smokers. Three patients $(33.3 \%)$ had received intravenous thrombolysis before endovascular treatment, and $6(66.6 \%)$ underwent endovascular treatment alone. Regarding the etiologies of the carotid occlusions, 7 patients $(77.7 \%)$ had atherosclerotic lesions, 1 patient $(11.1 \%)$ had an occlusive cardiac thrombus in the carotid bulb, and 1 patient $(11.1 \%)$ had a carotid dissection. Five patients $(55.5 \%)$ had a significant improvement in their baseline NIHSS scores $(\geq 4)$ from admission to discharge. Tables 1 and 2 summarize the aggregated and individual data, respectively, on the study sample. Carotid artery recanalization was achieved in 5 patients $(55.5 \%)$. For patients $2,4,8$, and 9 , a carotid recanalization procedure was not technically feasible because it was impossible to cross wires, balloons, or stents through an occluded carotid segment. An mTICI score of 3 was achieved in the 5 patients with successful recanalization, and no distal emboli were seen in the set of patients on whom recanalization had been attempted.

Patient 8 presented with a left occlusive carotid dissection associated with acute ischemic stroke symptoms (NIHSS score 7), and carotid recanalization was unsuccessful. This patient had a collateral contribution from the left posterior communicating artery (PCom) to the left carotid artery. Therefore, angioplasty and stenting of an ostial stenosis of $50 \%$ of the right dominant vertebral artery was performed, aiming to improve the intracranial circulation through the left PCom.

Patient 9 had a right carotid occlusion, and carotid recanalization was unsuccessful. This patient was admitted presenting with acute ischemic stroke (NIHSS score 8), and a bilateral stenosis of the V1 segment of the vertebral arteries was identified - 50\% in the right V1 and $80 \%$ in the left V1. Therefore, a bilateral vertebral angioplasty and stenting was performed to improve the intracranial collateral circulation through the right PCom. 
Table 1. Aggregated clinical and radiologic data on the patients $(n=9)$

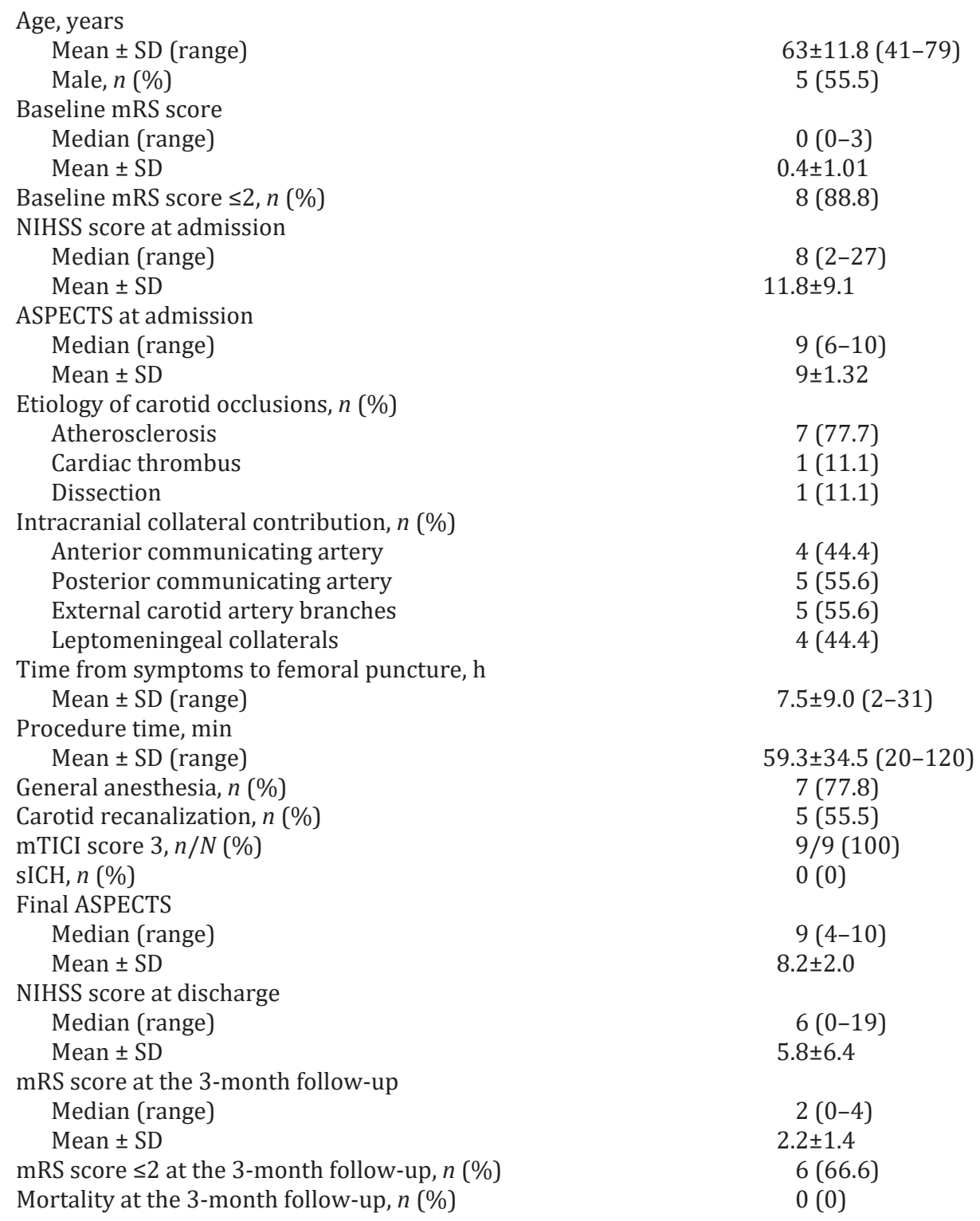

mRS, modified Rankin Scale; NIHSS, National Institutes of Health Stroke Scale; ASPECTS, Alberta Stroke Program Early CT Score; mTICI, modified Thrombolysis in Cerebral Infarction; sICH, symptomatic intracranial hemorrhage.

Both patient 8 and patient 9 showed clinical improvement after stenting, and their NIHSS scores improved from 7 and 8 at baseline to 1 and 1 at $24 \mathrm{~h}$ after treatment. No sICH or deaths were observed. Regarding neurologic outcomes at the 3-month follow-up, a good neurologic outcome (mRS score $\leq 2$ ) rate of $66.6 \%$ and no deaths were observed. Figures 1 and 2 illustrate the image assessment and endovascular treatment of patient 5 . Figures 3 and 4 illustrate the image assessment and endovascular treatment of patient 8. CT perfusion (software RAPID - iSchemaView) was performed on the last 4 patients included in the study, and all had a watershed ischemic core (see Fig. 1, 3). 
Table 2. Individual patient data

\begin{tabular}{|c|c|c|c|c|c|c|c|c|c|c|}
\hline $\begin{array}{l}\text { Pa- } \\
\text { tient } \\
\text { No. }\end{array}$ & $\begin{array}{l}\text { Age, } \\
\text { years/ } \\
\text { gender }\end{array}$ & $\begin{array}{l}\text { mRS } \\
\text { score at } \\
\text { baseline/ } \\
3 \text { months }\end{array}$ & $\begin{array}{l}\text { NIHSS score } \\
\text { at baseline/ } \\
\text { at discharge }\end{array}$ & $\begin{array}{l}\text { ASPECTS } \\
\text { at baseline/ } \\
\text { at discharge }\end{array}$ & $\begin{array}{l}\text { Etiology of carotid } \\
/ \text { occlusion } \\
\text { e }\end{array}$ & $\begin{array}{l}\text { Carotid occluded/ } \\
\text { segment }\end{array}$ & Collaterals & $\begin{array}{l}\text { Time from } \\
\text { symptom } \\
\text { onset to } \\
\text { puncture, h }\end{array}$ & $\begin{array}{l}\text { Procedure } \\
\text { time, min }\end{array}$ & $\begin{array}{l}\text { Carotid } \\
\text { recanali- } \\
\text { zation/ } \\
\text { mTICI score }\end{array}$ \\
\hline 1 & $67 / F$ & $1 / 3$ & $27 / 12$ & $10 / 8$ & Atherosclerosis & Left ICA/C1 & Acom & 3.5 & 50 & Yes/3 \\
\hline 2 & $79 / \mathrm{F}$ & $0 / 2$ & $9 / 7$ & $9 / 6$ & Atherosclerosis & Left ICA/C1-C2 & Acom, Pcom, ECA, LC & 5.5 & 70 & No/- \\
\hline 3 & $54 / \mathrm{M}$ & $0 / 2$ & $23 / 6$ & $10 / 10$ & Cardiac thrombus & Left ICA/C1 & Acom & 4 & 20 & Yes/3 \\
\hline 4 & $62 / \mathrm{M}$ & $0 / 1$ & $4 / 0$ & $9 / 9$ & Atherosclerosis & Right ICA/C1-C2 & ECA, LC & 4.1 & 120 & No/- \\
\hline 5 & $41 / \mathrm{M}$ & $0 / 2$ & $6 / 7$ & $8 / 8$ & Atherosclerosis & Left ICA/C1-C2 & Acom, Pcom, ECA & 10 & 45 & Yes/3 \\
\hline 6 & $70 / \mathrm{M}$ & $0 / 0$ & $2 / 0$ & $10 / 10$ & Atherosclerosis & Bilateral ICA/C1-C2 & ECA, LC & 31 & 32 & Yes/3 \\
\hline 7 & 78/M & $3 / 4$ & $21 / 19$ & $6 / 4$ & Atherosclerosis & Left ICA/C1-C2 & Acom, ECA & 4.9 & 37 & Yes/3 \\
\hline 8 & $59 / \mathrm{F}$ & $0 / 2$ & $7 / 1$ & $9 / 9$ & Dissection & Left ICA/C1-C2 & Pcom & 3 & 110 & No/- \\
\hline 9 & $63 / F$ & $0 / 1$ & $8 / 1$ & $10 / 10$ & Atherosclerosis & Right ICA/CI-C2 & Pcom, LC & 2 & 50 & No/- \\
\hline
\end{tabular}

mRS, modified Rankin Scale; NIHSS, National Institutes of Health Stroke Scale; ASPECTS, Alberta Stroke Program Early CT Score; mTICI, modified Thrombolysis in Cerebral Infarction; ICA, internal carotid artery; Acom, anterior communicating artery; Pcom, posterior communicating artery; ECA, external carotid artery; LC, left common carotid artery.
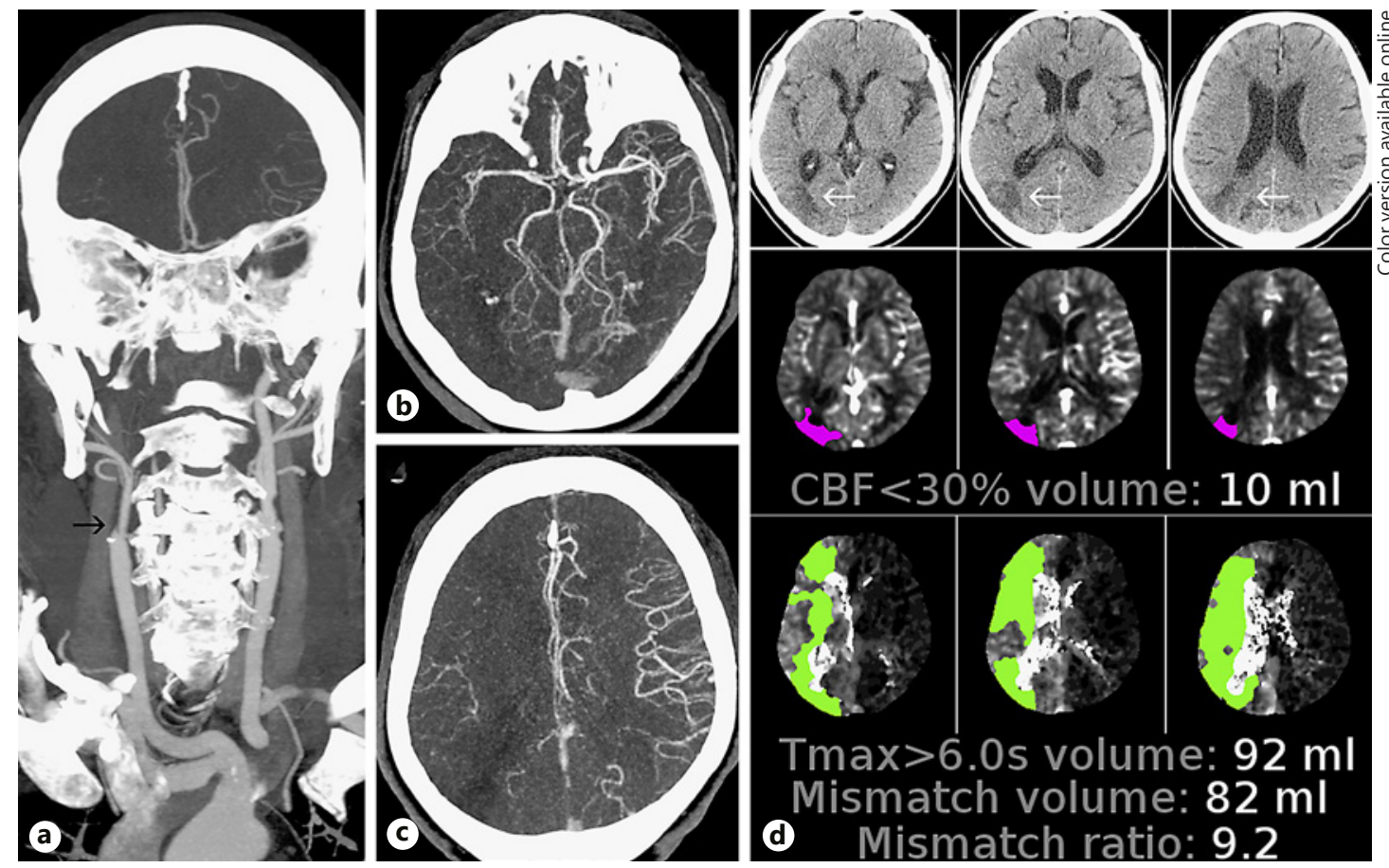

Fig. 1. Patient 5. a-c CT angiography. a Coronal view showing a right cervical internal carotid artery occlusion (black arrow). b, c Axial views showing no intracranial occlusion, a complete Willis polygon (b), and poor distal arterial vascularization to the right hemisphere (c). d Noncontrast CT scan of the brain at admission (ASPECTS 8) (white arrows) and CT perfusion using the RAPID platform (iSchemaView; http://www. i-rapid.com) showing a mismatch of the right hemisphere, defined as a volume mismatch of $82 \mathrm{~mL}$ and a mismatch ratio of 9.2 .

Among the patients who had no cervical carotid recanalization (patients 2, 5, 8, and 9), we performed vertebral angioplasty on patients 8 and 9, and both improved their NIHSS scores during hospitalization (see NIHSS scores in Table 2). Patient 2 had no fluctuating NIHSS scores but a small improvement from 9 to 7 during 5 days in hospital. Patient 5 had fluctuating NIHSS scores during 4 days in hospital (i.e., 6, 8, 5, and then 7). 

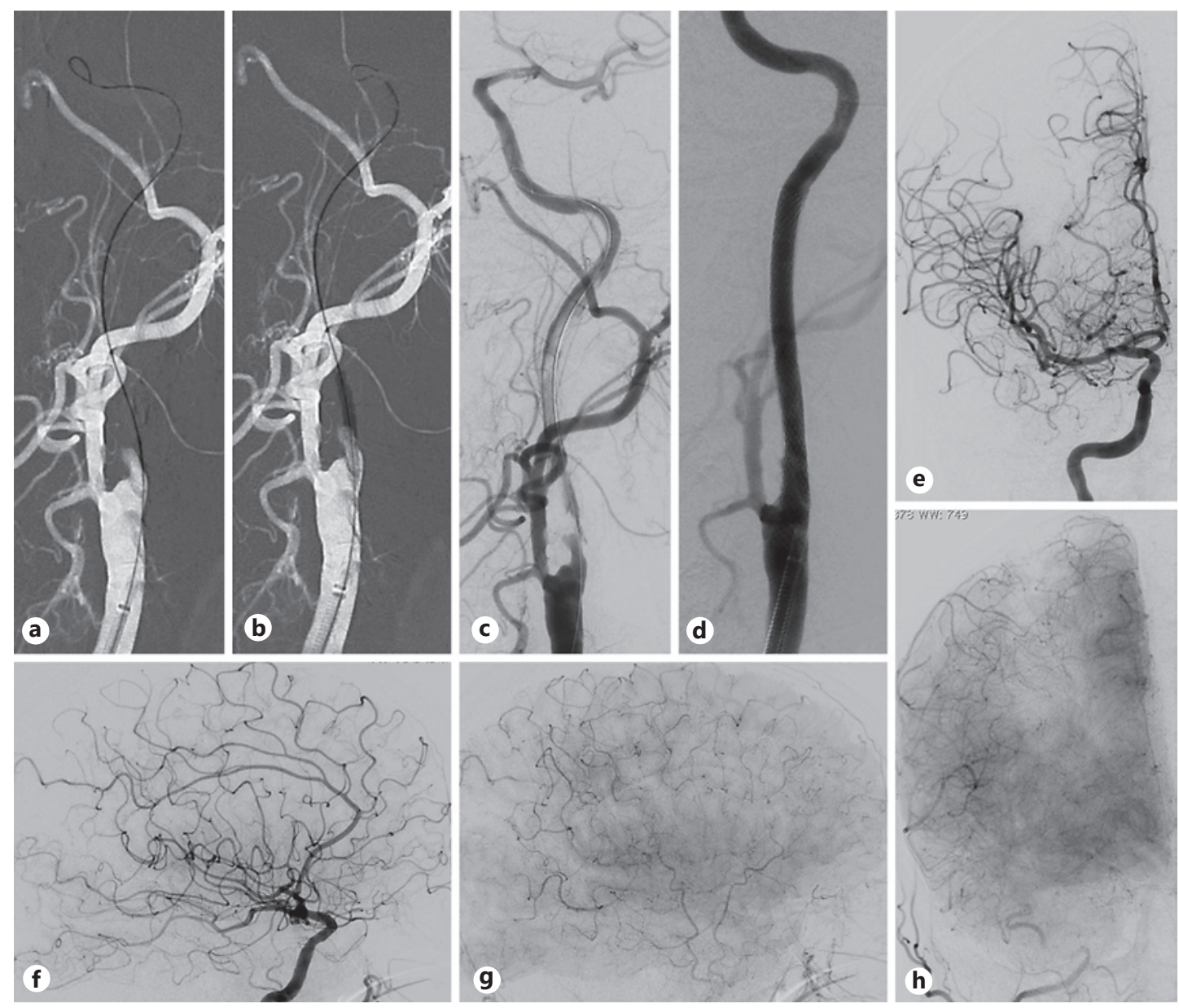

Fig. 2. Patient 5. a-d Digital subtraction angiograms of the right common carotid artery showing the steps of endovascular recanalization of an acute right internal carotid artery occlusion using angioplasty and stenting. e-h Subtraction angiograms of the brain, right common carotid injection. Frontal view: arterial (e) and capillary (h) phases; lateral view: arterial (f) and capillary (g) phases. The images demonstrate complete intracranial flow restoration to the right hemisphere.

Only patients 3,4 , and 8 had received intravenous r-tPA infusions before endovascular treatment. Among those 3 patients, carotid recanalization was achieved only in patient 3. Patient 3 had a carotid occlusion caused by a large cardiac thrombus. Despite r-tPA infusion, recanalization of the carotid was achieved only after stenting. Patients 4 and 8 had carotid occlusions caused by an atherosclerotic lesion and by a dissection, respectively. Both patient 4 and patient 8 had received intravenous r-tPA before endovascular treatment, but carotid recanalization was not achieved.

\section{Discussion}

In the present study, carotid tandem occlusions represented $17 \%$, while isolated cervical carotid occlusions represented $4.1 \%$, of all large vessel occlusions treated by thrombectomy. As demonstrated in this study, a true isolated cervical carotid occlusion, i.e., one that presents with no associated intracranial occlusion, may be infrequent in the setting of acute stroke 


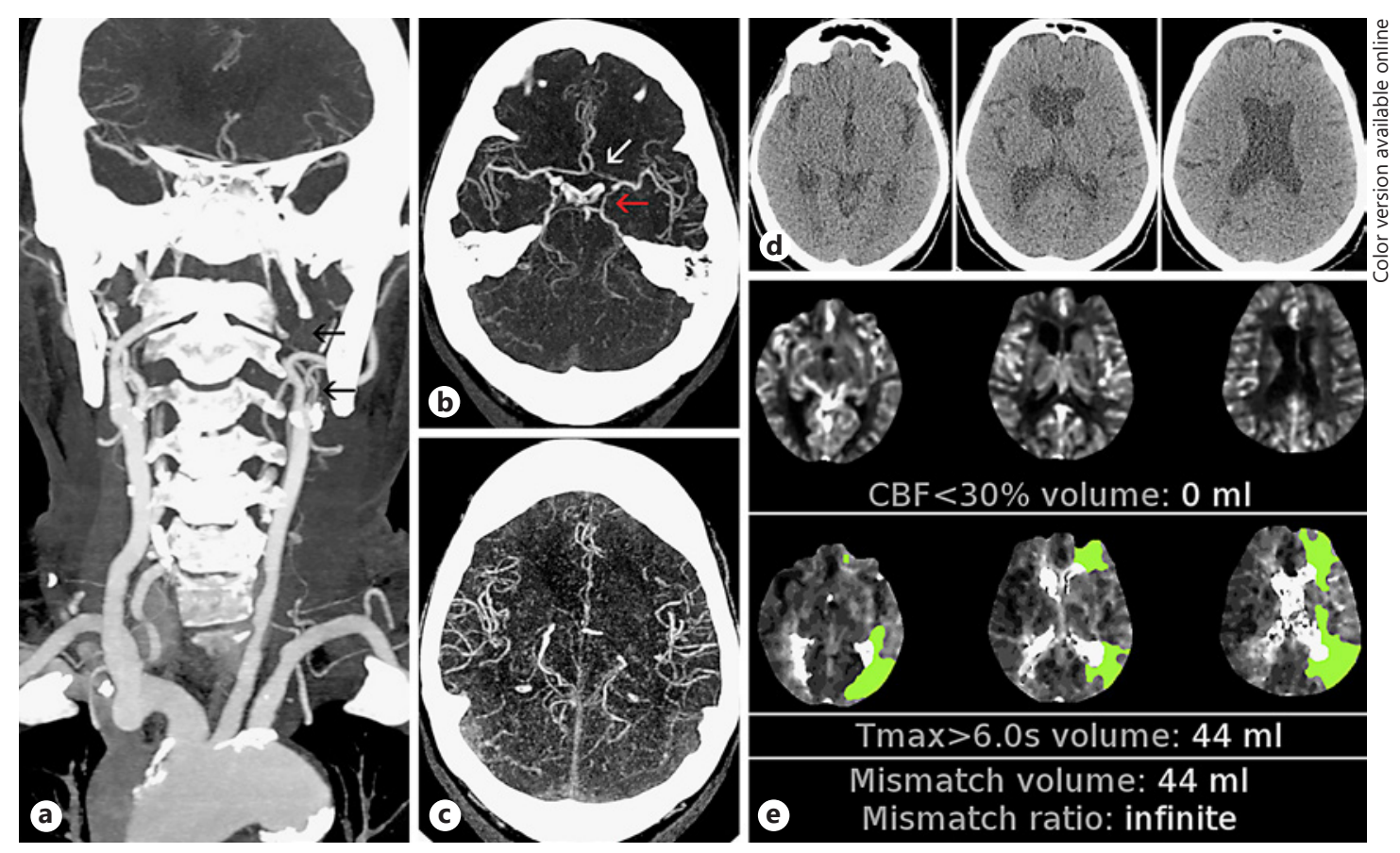

Fig. 3. Patient 8. a-c CT angiograms. a Coronal view showing a left cervical internal carotid occlusion (black arrows). b, c Axial views showing no intracranial occlusion, hypoplasia of the left A1 segment (b; white arrow) and a large left posterior communicating artery (red arrow), and symmetry between the two hemispheres regarding distal intracranial arterial vascularization (c). d Noncontrast CT scan of the brain at admission (ASPECTS 9). e CT perfusion using the RAPID platform showing no infarct core and watershed hypoperfusion of the left hemisphere (green), resulting in a mismatch volume of $44 \mathrm{~mL}$.

treatment. Few studies reporting results on the acute treatment of cervical carotid occlusions art to be found in the literature, and they have shown conflicting methods and results $[9,10$, 13-18]. Failure to provide a clear definition of carotid occlusions and the imaging method used to characterize the exact carotid segments that are occluded is an important limitation of previous studies. Most of the previous studies reporting results on acute stroke due to carotid lesions failed to provide a clear definition of what a carotid occlusion would be, and the studies may have included all patients presenting with high-grade stenosis, near occlusions, or subocclusions in a single "carotid occlusion" group. Studies that used Doppler may have confounded the detection of associated distal or intracranial occlusions. In contrast, studies reporting results regarding the endovascular treatment of acute carotid occlusions that used CT angiography scans or digital subtraction angiographic examinations exhibited greater accuracy in the detection of both extra- and intracranial occlusions [9, 10, 13-16].

The ICAR0-3 study compared the results of intravenous thrombolysis and endovascular treatment of patients with acute stroke associated with carotid occlusions [10]. The authors showed that endovascular treatment of patients with acute internal carotid occlusion resulted in a higher rate of intracranial bleeding, a similar rate of good functional outcome, and a lower mortality rate than treatment with intravenous thrombolysis. A total of 324 patients presenting with acute ischemic stroke due to extracranial carotid occlusion were included in the endovascular group. Forty-three patients (13.2\%) had carotid tandem occlusions, and the other 281 patients $(86.8 \%)$ had isolated extracranial carotid occlusions. Although all 324 patients underwent digital angiography, the authors did not provide a clear definition of what 

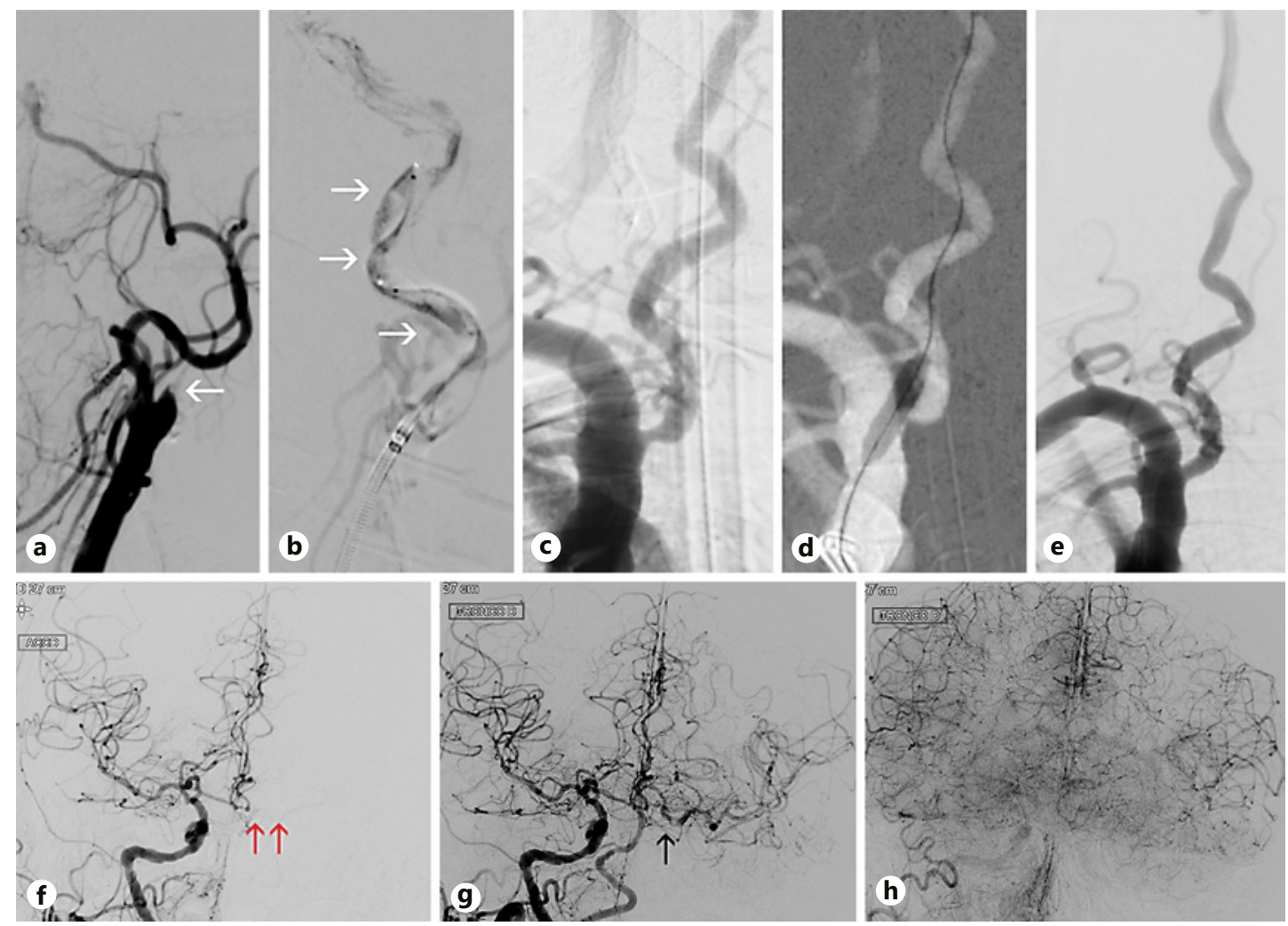

Fig. 4. Patient 8. a Digital subtraction angiogram (DSA) of the left common carotid artery (CCA) showing occlusion right after the internal carotid artery (ICA) bulb suggesting an arterial dissection (white arrow). b DSA of a microcatheter showing extensive occlusive dissection of the entire C2 segment of the left ICA during failed attempts at recanalization of the ICA (white arrows). c DSA frontal view showing stenosis of the origin of the right dominant vertebral artery (VA). d Roadmap of the right VA showing angioplasty with stenting of the right VA stenosis. e DSA after angioplasty stenting of the right VA. $\mathbf{f}$ DSA of the right CCA showing no absence of circulation from the anterior communicating artery to the left internal carotid territory (arrows). $\mathbf{g}, \mathbf{h}$ DSA of the brachiocephalic trunk after right VA angioplasty stenting. $\mathbf{g}$ Arterial phase. $\mathbf{h}$ Capillary phase. The images show collateral circulation to the left ICA territory through the left posterior communicating artery (black arrow).

they termed "extra-cranial carotid occlusions." Some patients may not have had complete carotid occlusions but carotid stenosis or carotid near-occlusions, and they were not reported in the study. Another limitation of the ICARO-3 study is the broad range of endovascular treatment strategies performed. Different combinations of intra-arterial thrombolysis, angioplasty, and stenting were reported, which limits data analysis and makes it difficult to assess the technical aspects of endovascular treatment [10].

In a review by Mokin et al. [9], the authors compared the results of intravenous thrombolysis and endovascular treatment of patients with acute stroke associated with carotid occlusions. The authors included 193 patients in an endovascular group and showed significantly higher rates of favorable outcomes ( 43.5 vs. $26.3 \% ; p=0.0001$ ) and of sICH (11.4 vs. $3.9 \% ; p=0.0011$ ) but not of mortality (26.4 vs. $27.2 \% ; p=0.85$ ) in the endovascular group compared with an intravenous thrombolysis group [8]. In a recent study, Jadhav et al. [17] included 107 acute symptomatic patients presenting with isolated proximal carotid occlusions that were treated by the endovascular approach. The authors 
showed a recanalization rate of $92 \%$ and a rate of good neurologic outcome of $65 \%$ (mRS score $\leq 2$ ), but distal embolization was observed in $22 \%$ of the patients, of whom $16 \%$ required intra-arterial treatment.

In the present study, we obtained a relatively high rate of favorable outcomes at the 3 -month follow-up (66.7\%), a rate of early neurologic improvement (NIHSS score improvement $\geq 4$ points) of $55.5 \%$, no sICH, and no deaths. The mean time from symptoms to femoral puncture was relatively long $(7.5 \mathrm{~h})$, and we included only patients presenting with total and isolated carotid occlusions at the level of the C1 or C1 and C2 segments. We used only angioplasty and stenting for carotid revascularization, independent of the etiology of the carotid occlusion (atherosclerosis, thrombus, dissection, or a combination of etiologies). In 4 patients (44\%), carotid recanalization was not achieved, probably because of the large extension of the occlusions or because of the chronicity of the occlusions. We may have treated some chronic or subacute carotid occlusions presenting with acute neurological symptoms. In this setting, clinical measures such as augmenting blood pressure and lying with the head flat have been indicated in stroke units, but the results of these practices are unpredictable and may not be sufficient in the long term. Therefore, we indicated endovascular treatment of carotid occlusions independent of the type of occlusion. When we compared the data on our 4 patients that did not achieve recanalization with the data from a new study [18] that showed a low recanalization rate of chronic carotid occlusion types $C$ and D, we had 3 patients with carotid occlusion type D (patients 2, 4, and 9) and 1 patient (patient 8) with a dissection. This finding may explain why we could not achieve carotid recanalization in those 3 patients.

In this study, we indicated thrombectomy for some patients presenting with low baseline NIHSS scores $(<6)$ (patients 4 and 6), which is currently a controversial decision. Patient 4 , with a baseline NIHSS score of 4, received intravenous r-tPA and carotid recanalization was not achieved. Patient 6 , with a baseline NIHSS score of 2 , did not receive intravenous r-tPA and carotid recanalization was achieved. Our decision to indicate endovascular treatment for these "low-NIHSS score patients" was guided by the CT perfusion results (RAPID software). We indicated endovascular treatment for those patients with a mismatch ratio $>2$ and a watershed pattern in $\mathrm{T}_{\max }$.

In this study, the recanalization of the vertebral stenosis in patients 8 and 9 improved their intracranial arterial flows through the PCom, resulting in immediate neurologic improvement after stenting. For some patients presenting with acute ischemic stroke due to extracranial occlusions, when artery recanalization was not possible, stenting the stenosis of other extracranial arteries (i.e., the contralateral carotid artery or dominant vertebral artery) in an attempt to improve intracranial collaterals appeared to be a reasonable strategy, although we cannot be certain whether these patients might have improved independent of stenting.

This study has considerable limitations because of its small sample size and the lack of a control group. The rate of $4.1 \%$ of patients with isolated carotid occlusions does not represent the real incidence of carotid occlusions at our stroke unit during the period of the study, because we included only patients who underwent endovascular treatment; patients that were only clinically managed were not included in this study. Thus, we cannot estimate the incidence of isolated cervical carotid occlusions at our stroke unit during the period of this study. Because of our small sample, some complications like sICH, which usually range in incidence between 3 and $8 \%$, may be absent, and a larger series could reveal such infrequent complications. In addition to that, other reasons that may explain our zero rate of sICH could be lower baseline NIHSS scores and the absence of intracranial device passes. The limitations described above may reduce the generalizability of the present study. Large controlled studies are necessary to confirm our findings. 


\section{Conclusions}

The endovascular recanalization of isolated cervical carotid occlusions presenting with acute ischemic stroke symptoms is feasible. Because isolated cervical carotid occlusions are associated with hemodynamic ischemic symptoms, if carotid recanalization cannot be achieved, stenting other cervical arteries' stenoses, with a focus on intracranial flow improvement, appears to be a reasonable strategy. Large controlled studies are necessary to assess the safety and efficacy of recanalization of acute isolated cervical carotid occlusions.

\section{Statement of Ethics}

This study was approved by the ethics committee of our institution, and the review board waived the need for written informed consent from the participants.

\section{Disclosure Statement}

On behalf of all authors, the corresponding author states that there is no conflict of interest.

\section{References}

1 Smith WS, Lev MH, English JD, Camargo EC, Chou M, Johnston SC, et al. Significance of large vessel intracranial occlusion causing acute ischemic stroke and TIA. Stroke. 2009 Dec;40(12):3834-40.

2 Goyal M, Demchuk AM, Menon BK, Eesa M, Rempel JL, Thornton J, et al.; ESCAPE Trial Investigators. Randomized assessment of rapid endovascular treatment of ischemic stroke. N Engl J Med. 2015 Mar;372(11):1019-30.

3 Campbell BC, Mitchell PJ, Kleinig TJ, Dewey HM, Churilov L, Yassi N, et al.; EXTEND-IA Investigators. Endovascular therapy for ischemic stroke with perfusion-imaging selection. N Engl J Med. 2015 Mar;372(11):1009-18.

4 Berkhemer OA, Fransen PS, Beumer D, van den Berg LA, Lingsma HF, Yoo AJ, et al.; MR CLEAN Investigators. A randomized trial of intraarterial treatment for acute ischemic stroke. N Engl J Med. 2015 Jan;372(1):11-20.

5 Saver JL, Goyal M, Bonafe A, Diener HC, Levy EI, Pereira VM, et al.; SWIFT PRIME Investigators. Stent-retriever thrombectomy after intravenous t-PA vs. t-PA alone in stroke. N Engl J Med. 2015 Jun;372(24):2285-95.

6 Jovin TG, Chamorro A, Cobo E, de Miquel MA, Molina CA, Rovira A, et al.; REVASCAT Trial Investigators. Thrombectomy within 8 hours after symptom onset in ischemic stroke. N Engl J Med. 2015 Jun;372(24):2296-306.

7 Goyal M, Menon BK, van Zwam WH, Dippel DW, Mitchell PJ, Demchuk AM, et al.; HERMES collaborators. Endovascular thrombectomy after large-vessel ischaemic stroke: a meta-analysis of individual patient data from five randomised trials. Lancet. 2016 Apr;387(10029):1723-31.

8 Campbell BC, Hill MD, Rubiera M, Menon BK, Demchuk A, Donnan GA, et al. Safety and efficacy of solitaire stent thrombectomy: individual patient data meta-analysis of randomized trials. Stroke. 2016 Mar;47(3):798-806.

9 Mokin M, Kass-Hout T, Kass-Hout O, Dumont TM, Kan P, Snyder KV, et al. Intravenous thrombolysis and endovascular therapy for acute ischemic stroke with internal carotid artery occlusion: a systematic review of clinical outcomes. Stroke. 2012 Sep;43(9):2362-8.

10 Paciaroni M, Inzitari D, Agnelli G, Caso V, Balucani C, Grotta JC, et al. Intravenous thrombolysis or endovascular therapy for acute ischemic stroke associated with cervical internal carotid artery occlusion: the ICARO-3 study. J Neurol. 2015 Feb;262(2):459-68.

11 Pontes-Neto OM, Cougo P, Martins SC, Abud DG, Nogueira RG, Miranda M, et al. Brazilian guidelines for endovascular treatment of patients with acute ischemic stroke. Arq Neuropsiquiatr. 2017 Jan;75(1):50-6.

12 Wahlgren N, Ahmed N, Dávalos A, Ford GA, Grond M, Hacke W, et al.; SITS-MOST investigators. Thrombolysis with alteplase for acute ischaemic stroke in the Safe Implementation of Thrombolysis in Stroke-Monitoring Study (SITS-MOST): an observational study. Lancet. 2007 Jan;369(9558):275-82.

13 Yeo LL, Kong WY, Paliwal P, Teoh HL, Seet RC, Soon D, et al. Intravenous Thrombolysis for Acute Ischemic Stroke due to Cervical Internal Carotid Artery Occlusion. J Stroke Cerebrovasc Dis. 2016 Oct;25(10):2423-9.

14 Endo S, Kuwayama N, Hirashima Y, Akai T, Nishijima M, Takaku A. Results of urgent thrombolysis in patients with major stroke and atherothrombotic occlusion of the cervical internal carotid artery. AJNR Am J Neuroradiol. 1998 Jun-Jul;19(6):1169-75.

15 Paciaroni M, Balucani C, Agnelli G, Caso V, Silvestrelli G, Grotta JC, et al. Systemic thrombolysis in patients with acute ischemic stroke and Internal Carotid ARtery Occlusion: the ICARO study. Stroke. 2012 Jan;43(1):12530 . 
16 De Silva DA, Brekenfeld C, Ebinger M, Christensen S, Barber PA, Butcher KS, et al.; Echoplanar Imaging Thrombolytic Evaluation Trial (EPITHET) Investigators. The benefits of intravenous thrombolysis relate to the site of baseline arterial occlusion in the Echoplanar Imaging Thrombolytic Evaluation Trial (EPITHET). Stroke. 2010 Feb;41(2):295-9.

17 Jadhav A, Panczykowski D, Jumaa M, Aghaebrahim A, Ranginani M, Nguyen F, et al. Angioplasty and stenting for symptomatic extracranial non-tandem internal carotid arteryocclusion. J Neurointerv Surg. 2018 Apr;neurintsurg-2018-013810. Epub ahead of print.

18 Hasan D, Zanaty M, Starke RM, Atallah E, Chalouhi N, Jabbour P, et al. Feasibility, safety, and changes in systolic blood pressure associated with endovascular revascularization of symptomatic and chronically occluded cervical internal carotid artery using a newly suggested radiographic classification of chronically occluded cervical internal carotid artery: pilot study. J Neurosurg. 2018 May. doi: 10.3171/2018.1.JNS172858. Epub ahead of print. 